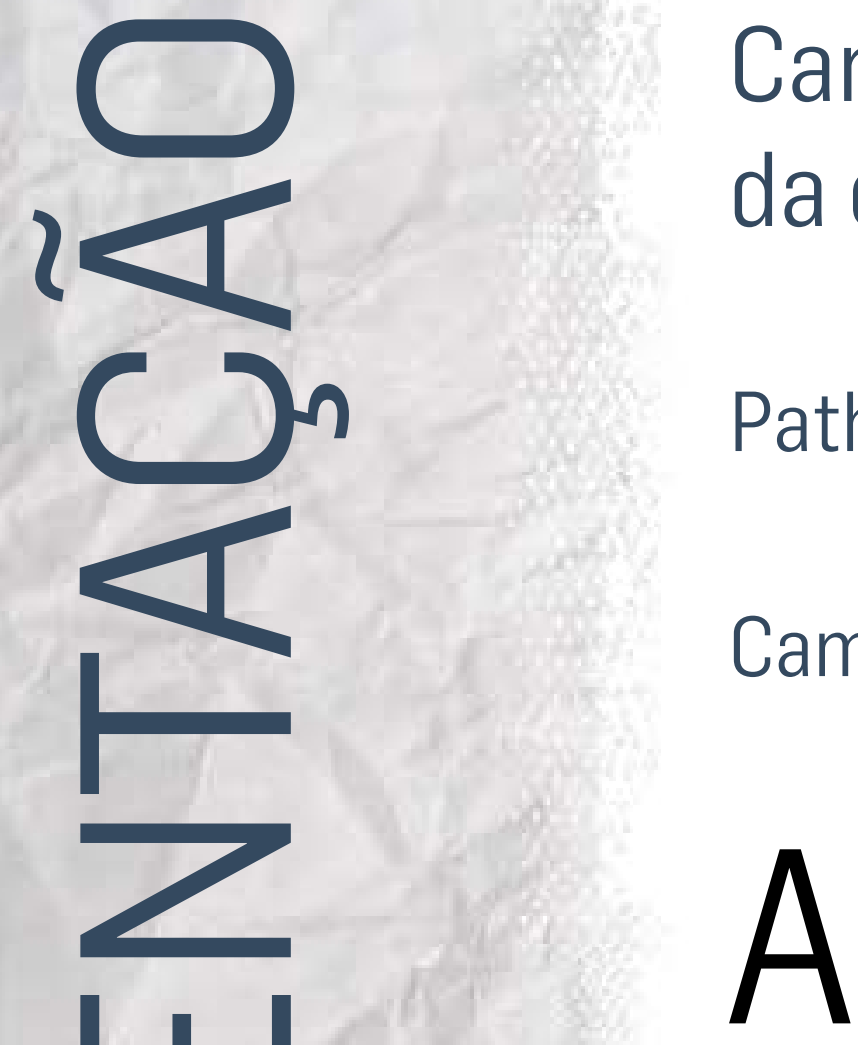

\title{
Caminhos para a prática da excelência
}

\section{Paths for the practice of excellence}

\section{Caminos para la práctica de la excelencia}

regiões brasileiras, em outras eles florescem, acompanhados de cursos de lato sensu e mesmo de mestrados e de doutorados - entre elas o de Interfaces da Comunicação, do Programa de Pós-Graduação da Escola de Comunicações e Artes da Universidade de São Paulo (PPGCom/ ECA-USP) - que apresentam linhas de pesquisa nas quais temáticas de relações públicas e de áreas afins são devidamente abrigadas.

Isso significa que ícones já podem ser reconhecidos nos âmbitos acadêmico e profissional, que as discussões em torno do assunto crescem em número e qualidade. Por causa disso - e por questão óbvia de sua razão de existência - Organicom traz neste dossiê pesquisas que abordam as dimensões e as práticas do campo das relações públicas e da comunicação organizacional no Brasil e também no exterior. Leve-se em consideração que os níveis e números das dimensões e das práticas em relações públicas também apresentam significativas variações em relação aos diversos países: alguns já têm claros níveis de excelência, com mercado estruturado e pesquisas que the deem suporte, outros por vezes têm maior força em um aspecto que o outro.

Quando demos início ao trabalho de Organicom, há mais de uma década, já havia motivos para que comemorássemos 0 amadurecimento da área, especialmente em nosso país. Desde então, cada um de nossos dossiês marcou um tema emergente que dava ao campo mais amplitude e possibilidades de amadurecimento: comunicação e mudança cultural nas organizações; avaliação e mensuração em comunicação organizacional; comunicação 
digital; comunicação pública e governamental; relações públicas: campo acadêmico e profissional; comunicação de risco e crise: prevenção e gerenciamento; identidade de marca e gestão da comunicação corporativa; ética e comunicação nas organizações; discurso institucional, linguagem e retórica; comunicação organizacional e relações públicas: pesquisa, reprodução, aplicação; ouvidoria e comunicação; comunicação, marketing e produção cultural; lobby, relações governamentais, democracia; comunicação e esporte: pesquisa, marketing e mídia; comunicação e saúde; comunicação e sustentabilidade; comunicação interna: processos e as interações nas organizações; memória institucional, narrativas e storytelling; interculturalidade e organizações; comunicação e economia criativa; redes sociais: usos corporativos; e, no dossiê deste número 24, "Relações públicas: dimensões e práticas".

Como Elizabeth Saad Corrêa e Luiz Alberto de Farias diziam na apresentação de nosso número anterior, ainda se discute 0 protagonismo da atividade de comunicação nas organizações, em um momento no qual as tecnologias digitais de informação e comunicação (TICS) impactam fortemente o fazer e o pensar comunicativos. E esse fazer-pensar é por natureza e definição complexo e permite a conexão com diversos outros pensamentos, além de estar presente em todas as atividades humanas. Com a maior necessidade de colaboração e de valorização do pensamento baseado na tolerância, mais e mais discussões devem ser feitas sobre o papel das relações públicas na harmonização de interesses.

A presente edição traz uma ampla rede de discussões na seção "Espaço aberto". Esta inicia com o artigo "Marcas organizacionais e o patrocínio de causas sociais alternativas: o daltonismo", produzido a partir de pesquisa de mestrado desenvolvida por Gustavo Henrique Del Vechio, sob a orientação de Kleber Markus. Em outra frente, Ana Paula da Rosa e Bruno Garcia Vinhola oferecem o texto "Entre a disputa e a coprodução: a imagem do Exército Brasileiro no Complexo da Maré". Terezinha Silva apresenta o artigo "O acontecimento no contexto das organizações: a campanha "Casais", os públicos e a imagem de 0 Boticário". E Angela Cristina Salgueiro Marques e Frederico da Cruz Vieira de Souza trazem o texto "Ultraje do rosto: embates discursivos e reconhecimento da liderança feminina na Petrobras".

A essa seção seguem os artigos que consolidam o dossiê "Relações públicas: dimensões e práticas". Lucie Gerber e Krishnamurthy Sriramesh trazem o artigo "Corporate social responsibility in the Swiss watch industry: perceptions and practices"; Maria-Rosa Collell e Jordi Xifra, o artigo "The recent history of public relations in Spain"; com o texto "Social neuroscience: new approaches for public relations and communications management", o canadense Terry Flynn discute uma nova abordagem para as relações públicas. Ainda no olhar internacional desta edição, Alice Zozima Rego de Souza Paris e Larissa Conceição dos Santos apresentam o artigo "Comunicação organizacional à la française: avanços e perspectivas"; o pesquisador Guilherme de Fráguas Nobre discute o tema "Diplomacia de negócios e relações públicas internacionais"; Erica de Castro Correa e Juan-Carlos Molleda trazem "Using indigenous culture to create brand identities of transnational corporations from developing countries". Por fim, Ana Maria Dantas de Maio apresenta o artigo "Comunicação face a face nas organizações em tempos de sociedade midiatizada"; e Paulo Nassar, Mateus Furlanetto e Luiz-Alberto de Farias trazem o artigo "Cenário histórico das relações públicas no Brasil".

São pontos de destaque nesta edição duas entrevistas com profissionais de grande relevância: um representando o mercado, outro a academia, sem querermos distinguir ou hierarquizar esses dois espaços; ao contrario, procurando exatamente mostrar a proximidade essencial entre ambos. Valéria de Siqueira Castro Lopes, Paulo Nassar e Luiz Alberto de Farias conversaram com o pesquisador norte-americano, especialista em relações com investidores e relações públicas financeiras, Alexander Laskin, da Universidade Quinnipiac, em Connecticut, gerando a entrevista "Relações públicas: tanto ainda a ser explorado!", que aborda as perspectivas para a área a partir da visão norte-americana. Já Nara Almeida, Paulo Nassar e Luiz Alberto 
de Farias puderam ouvir Richard Edelman, CEO de um dos maiores conglomerados de comunicação e relações públicas do mundo, resultando na entrevista "Comunicador: o Davi de todos os Golias", na qual Edelman mostra o momento importante pelo qual passa a área de comunicação e de relações públicas e como ela ganha importância e espaço.

No perfil desta edição optamos por escolher cinco pesquisas para adensar a discussão sobre a realidade das relações públicas: "El manejo de las relaciones públicas internacionales en la crisis de Volkswagen", de Amaya Arribas; "Comunicação comunitária e responsabilidade social em clubes de futebol do Brasil e da Europa: muito além do "sócio-torcedor'", de Ary José Rocco Júnior, Eduardo de Oliveira Cruz Carlassara e Pedro Lucas Leite Parolini; "Inovação na comunicação da memória institucional: caso dos 100 anos da CPFL Energia", de João de Deus Dias Neto e Priscila Ferreira Perazzo; "Marca Rio: uma grife além da cidade", de Patrícia Cerqueira Reis, e "Comunicación política y movimientos sociales en España: del 15M a Podemos", de Ana Almansa-Martínez e María Jesús Fernández Torrres.

Por fim, Organicom apresenta as resenhas dos livros Storytelling: as narrativas da memoria na estratégia de comunicação, de Rodrigo Silveira Cogo, resenhado por Emiliana Pomarico Ribeiro; e Comunicação organizacional estratégica: aportes conceituais e aplicados, organizada por Margarida M. Krohling Kunsch, resenhada por Ana Claudia Pompeu Torezan Andreucci.

Já nos ensinava Ortega y Gasset, em Rebelião das massas (1937), que nós somos nós e as nossas circunstancias, donde a necessidade de nos mantermos atentos aos movimentos do campo, da evolução que pode ser acompanhada à medida que haja trabalhos que façam esse monitoramento. Relações públicas e comunicação organizacional hoje ganham espaço em pesquisas e práticas, ampliando sua participação nas estratégias das organizações - em termos de budgete de participação no planejamento. 